\title{
Phenotype of mesenchymal stem cells from patients with myelodyplastic syndrome maybe partly modulated by decitabine
}

\author{
YANBIN PANG ${ }^{1-4^{*}}$, SUXIA GENG ${ }^{2,3^{*}}$, HONGYANG ZHANG ${ }^{1-3}$, PEILONG LAI $^{2,3}$, PENGJUN LIAO $^{2,3}$, \\ LINGJI ZENG ${ }^{2,3}$, ZESHENG LU ${ }^{2,3}$, JIANYU WENG ${ }^{1-3,5}$ and XIN DU ${ }^{1-3,5}$ \\ ${ }^{1}$ The Second School of Clinical Medical, Southern Medical University, Guangzhou, Guangdong 510515; \\ ${ }^{2}$ Department of Hematology, Guangdong Provincial People's Hospital and Guangdong Academy of Medical Sciences; \\ ${ }^{3}$ Department of Hematology, Guangdong Geriatrics Institute, Guangzhou, Guangdong 510080; \\ ${ }^{4}$ Department of Hematology, Affiliated Hospital of Hebei University, Baoding, Hebei 071000; ${ }^{5}$ Department of \\ Hematology, South China University of Technology School of Medicine, Guangzhou, Guangdong 5100065, P.R. China
}

Received March 30, 2018; Accepted June 25, 2019

\section{DOI: $10.3892 / \mathrm{ol} .2019 .10788$}

\begin{abstract}
Mesenchymal stem cells (MSCs) derived from myelodysplastic syndromes (MDSs) have been demonstrated to accelerate the progression of MDS. However, whether the phenotype of MSCs derived from MDS (MDS-MSCs) may be reversed and serve as a potential target for the treatment of MDS remains unclear. The present study investigated the functional alternations of MDS-MSCs following in vitro decitabine-treatment. Primary MSCs were cultured from the bone marrow aspirates of 28 patients with MDS. The impact on the growth of MDS-MSCs treated with decitabine was analyzed using the MTT assay. Changes in the gene expression levels of runt related transcription factor 2 (RUNX2), Sp7 transcription factor (SP7), cyclin dependent kinase inhibitor 1A (CDKN1A) and CD274 in MDS-MSCs following treatment with decitabine were analyzed by reverse transcription-quantitative polymerase chain reaction. The effects of decitabine on apoptosis and the cell cycle were examined using flow cytometry. The effect of decitabine on the immune regulation of MDS-MSCs was tested by the co-culture of MSCs with activated T cells in vitro. The results revealed that proliferation, apoptosis and the mRNA expression levels of RUNX2 and SP7 in MDS-MSCs did not significantly change following treatment with decitabine compared with control MDS-MSCs. However, treatment with decitabine resulted in a smaller population of cells in the G1 phase and an increase in the number of cells
\end{abstract}

Correspondence to: Dr Xin Du, Department of Hematology, Guangdong Provincial People's Hospital and Guangdong Academy of Medical Sciences, 106 Zhongshan Er Road, Guangzhou, Guangdong 510080, P.R. China

E-mail: miyadu@hotmail.com

*Contributed equally

Key words: myelodysplastic syndrome, mesenchymal stem cells, cell cycle, regulatory $\mathrm{T}$ cells in the G2/M phase compared with control MDS-MSCs. This change was associated with decreased expression of CDKN1A in cells treated with decitabine compared with control cells. Notably, the ability of MDS-MSCs treated with decitabine to induce the differentiation of $\mathrm{T}$ cells into regulatory $\mathrm{T}$ cells was significantly reduced compared with control MDS-MSCs. This was associated with a decreased expression of CD274 in MDS-MSCs treated with decitabine compared with control MDS-MSCs. In conclusion, the phenotype of MSCs derived from patients with MDS was partially reversed by treatment with decitabine, presenting a potential therapeutic intervention.

\section{Introduction}

Myelodysplastic syndromes (MDSs) are a diverse group of clonal diseases derived from hematopoietic stem cells (HSCs), which are characterized by ineffective hematopoiesis, progressive bone marrow (BM) failure and a substantial risk of progression to acute myeloid leukemia (AML) $(1,2)$. Although the majority of previous studies have focused on the molecular and genetic changes in malignant hematopoietic cells, the mechanisms underlying the progression from less advanced MDS, with dysplastic progenitors and apoptosis in the bone marrow, to advanced MDS, with an excess of blasts, have not been elucidated $(3,4)$. Accumulated evidence in recent years has demonstrated that MDS is a complex disease that involves abnormalities in HSCs and the bone marrow microenvironment (4-6).

Mesenchymal stem cells (MSCs), which possess the ability of self-renewal and multiple differentiation potential, serve important roles in the support of hematopoiesis and maintaining immune homeostasis $(7,8)$. The deletion of dicer 1 , ribonuclease III in the mesenchymal lineage induced an MDS-like phenotype, while the deletion of SBDS ribosome maturation factor in MSCs induced genotoxic stress through inflammatory tumor protein p53 (TP53)-S100 calcium binding protein A8/9-TLR4 signaling in animal models $(5,6)$. In addition, MSCs derived from patients with MDS are required for the propagation of MDS-initiating Lin ${ }^{-C D} 34{ }^{+} \mathrm{CD} 38$ stem cells in orthotropic xenografts (4). Although knowledge about 
the role of MSCs in patients with MDS is limited, studies have revealed that MSCs derived from patients with MDS (MDS-MSCs) exhibited a senescent phenotype, which included decreased proliferation and differentiation potential and the production of secreted factors, such as jagged-1, C-C motif chemokine ligand 3 and vascular endothelial growth factor, which promote the progression of MDS (4,9-11). Senescence in MDS-MSCs that do not have shortened telomeres may be induced by the upregulation of the mitogen-activated protein kinase 14/mitogen activated protein kinase/CDKN1A signaling pathway and the abnormal expression of microRNAs. The blocking of these pathways successfully reversed senescence in MDS-MSCs $(9,12,13)$. MDS-MSCs may therefore provide a potential target for therapeutic interventions.

Decitabine (5-aza-2-deoxycytidine), an inhibitor of DNA methyltransferases, has a wide range of anticancer activities (14). Previous studies suggested that the differentiation and immunomodulatory function of MSCs maybe regulated by decitabine $(15,16)$. However, whether this applies to MDS-MSCs and whether it has potential clinical significance remains unknown. The current study isolated primary MSCs cultures from the bone marrow of patients with different MDS subtypes and investigated the effects of decitabine on their proliferation, differentiation and immunomodulatory functions.

\section{Materials and methods}

Patients and samples. Bone marrow specimens were obtained from 40 patients with newly-diagnosed MDS (median age, 54 years; range, 24-80 years) at the Guangdong General Hospital between January 2014 and March 2015. According to the World Health Organization 2008 classification of MDS (17), 15 patients $(37.5 \%)$ had refractory cytopenia with multilineage dysplasia, 2 patients $(5.0 \%)$ had refractory anemia with ringed sideroblasts, 14 patients $(35.0 \%)$ had refractory anemia with excess blasts, 4 patients $(10 \%)$ had unclassified MDS and 2 patients $(5.0 \%)$ had AML with myelodysplasia-related changes (Table I). In addition, there were 3 patients (7.5\%) with chronic myelomonocytic leukemia. Primary MSCs cultures were successfully generated from 28 of these 40 patients. Written informed consent was obtained from all patients and donors, and the study was performed in accordance with the Declaration of Helsinki and approved by the Research Ethics Committee of Guangdong General Hospital, Guangdong Academy of Medical Sciences.

Isolation and culture of BM-MSCs. Mononuclear cells were isolated from fresh BM aspirates at the time of initial diagnosis by centrifugation at $600 \mathrm{xg}$ for $15 \mathrm{~min}$ at room temperature using Ficoll-PaquePlus density gradient (specific gravity 1.077 g/ml; Sigma Diagnostics, Inc., Livonia, MI, USA). Mononuclear cells (1-7×10 ${ }^{7}$ cells) were seeded and cultured in Human Mesenchymal Stem Cell Growth Medium supplemented with $10 \%$ fetal bovine serum (FBS), $2 \mathrm{mM}$ glutamine, $100 \mathrm{U} / \mathrm{ml}$ Penicillin-Streptomycin [Cyagen Biosciences (Guangzhou) Inc.] at $37^{\circ} \mathrm{C}$ with $5 \% \mathrm{CO}_{2}$ in a fully humidified atmosphere. After $72 \mathrm{~h}$, the culture medium was replaced and non-adherent cells were removed, thereafter medium was replaced twice a week. Upon reaching $>70-80 \%$ confluence, cells were detached with $0.25 \%$ trypsin-EDTA (Gibco; Thermo Fisher Scientific, Inc.) and were seeded at $1 \times 10^{4}$ cells $/ \mathrm{cm}^{2}$ in T-25 flasks. All experiments were performed using MSCs derived from passages (P) 2-4. To fulfill the criteria of the International Society for Cellular Therapy (18) and to exclude contamination of MSCs cultures by hematopoietic cells, MSCs were suspended in $50 \mu \mathrm{l} \mathrm{FC} \mathrm{block} \mathrm{(cat.} \mathrm{no.} \mathrm{564219;} \mathrm{1:100;}$ BD Biosciences) on ice for 10-15 min. MSCs were washed twice with PBS containing 2\% fetal bovine serum (Gibco; Thermo Fisher Scientific, Inc.) and were incubated for $30 \mathrm{~min}$ at room temperature with antibodies against CD34 (cat. no. 555821), CD45 (cat. no. 555482), CD73 (cat. no. 550257), CD166 (cat. no. 560903), CD90 (cat. no. 555595) and CD105 (cat. no. 560839). All antibodies were used at a dilution of 1:100 and purchased from BD Biosciences. Following incubation with the primary antibodies, cells were subsequently fixed with $2 \%$ paraformaldehyde $(\mathrm{w} / \mathrm{v})$ overnight at $4^{\circ} \mathrm{C}$, in the dark. The same-species, same-isotype non-specific antibodies (mouse $\mathrm{IgG}_{1}$, $\kappa$; cat. cat. no. 555749 for CD73 and CD166; mouse BALB/c IgG 1 , $\kappa$; cat. no. 555748 for CD90, CD34 and CD45; mouse BALB/c IgG 1 , ; cat. no. 554680 for CD105; all 1:100 and purchased from BD Biosciences) were used as negative controls, and were incubated for $30 \mathrm{~min}$ at room temperature. Cell analysis was performed using a flow cytometer and FlowJo software (version 10; FlowJo LLC).

MSCs differentiation. The differentiation potential of MSCs toward the osteogenic and adipogenic lineages was assessed. In brief, MSCs (P2) were induced for adipogenesis following culture in Human Mesenchymal Stem Cell Adipogenic Differentiation medium [ADM; Cyagen Biosciences (Guangzhou), Inc.] containing $1 \mathrm{mM}$ dexamethasone, $0.2 \mathrm{mM}$ rosiglitazone, $0.5 \mathrm{mM} 3$-isobutyl-1-methylxanthine, $0.01 \mathrm{mg} / \mathrm{ml}$ insulin and $10 \%$ FBS for 14 days. After $72 \mathrm{~h}$, the ADM was replaced with adipogenesis maintenance medium [AMM; Cyagen Biosciences (Guangzhou), Inc.] containing $0.01 \mathrm{mg} / \mathrm{ml}$ insulin and 10\% FBS in Dulbecco's modified Eagle's medium. The spent AMM was replaced with fresh AMM following $24 \mathrm{~h}$. The treatment was repeated three times to achieve full adipogenic differentiation and the adipocyte phenotype was determined by Oil Red $\mathrm{O}$ staining. In brief, cells were washed with PBS and fixed with $4 \%(\mathrm{w} / \mathrm{v})$ paraformaldehyde (Sigma-Aldrich; Merck KGaA) in PBS for $30 \mathrm{~min}$ at room temperature. After discarding the paraformaldehyde, cells were evenly covered with $0.5 \%$ Oil Red $\mathrm{O}$ working solution (prepared $15 \mathrm{~min}$ before use by adding, mixing and filtering 3 parts of Oil Red $\mathrm{O}$ stock solution to 2 parts of water) [Cyagen Biosciences (Guangzhou) Inc.] for $30 \mathrm{~min}$ at room temperature and washed twice with PBS. Cells were covered with water and observed under a light microscope and images were captured (magnification, x100). Lipid droplets appeared red. The osteogenic differentiation was performed as previously described (19), and the osteogenic phenotype was subsequently visualized by Alizarin red staining. In brief, cells were washed with PBS and fixed with $4 \%$ (w/v) paraformaldehyde (in PBS for $30 \mathrm{~min}$ at room temperature. Cells were stained with Alizarin Red [1\%w/v; Cyagen Biosciences (Guangzhou) Inc.] for $5 \mathrm{~min}$, then washed with distilled water, and finally air-dried at room temperature. Cells were covered with water and observed 
Table I. Clinical characteristics of patients with myelodysplastic syndromes.

\begin{tabular}{lcc}
\hline Characteristic & No. of patients & \% of patients \\
\hline Sex & & \\
Male & 29 & 72.5 \\
Female & 11 & 27.5 \\
Diagnosis & & \\
RCMD & 15 & 37.5 \\
RARS & 2 & 5.0 \\
RAEB-I & 9 & 22.5 \\
RAEB-II & 5 & 12.5 \\
CMML & 3 & 7.5 \\
MDS-U & 4 & 10.0 \\
sAML & 2 & 5.0 \\
IPSS & & \\
Low & 6 & 15.0 \\
Intermediate-1 & 25 & 62.5 \\
Intermediate-2 & 7 & 17.5 \\
High & 2 & 5.0 \\
\hline
\end{tabular}

RCMD, refractory cytopenia with multilineage dysplasia; RARS, refractory anaemia with ring sideroblasts; RAEB, refractory anemia with excess blasts; CMML, chronic myelomonocytic leukemia; MDS-U, myelodysplastic syndrome-unclassified; sAML, acute myeloid leukemia with myelodysplasia-related changes; IPSS, international prognostic scoring system.

under a microscope. Images were captured (Olympus, IX70, Japan) at the time of observation. Mineralized matrix deposition appeared red.

Decitabine treatment. A previous study revealed that the antitumor effect of $0.02-0.30 \mu \mathrm{M}$ decitabine in vitro corresponded with the concentration of the clinically administered dose of decitabine and did not cause immediate cytotoxicity in primary leukemic and epithelial tumor cells in vitro (20). A previous study revealed that a concentration of decitabine $<10 \mu \mathrm{M}$ did not inhibit the proliferation of MDS-MSCs, and $0.25 \mu \mathrm{M}$ decitabine induced the immune response and enhanced the sensitivity of tumor cells to immune cells in vitro (21). Decitabine is generally administered daily for 5 consecutive days in a clinical setting (22). Therefore, the current study analyzed the effect of decitabine on MDS-MSCs for 5 days. Decitabine was dissolved in PBS ( $\mathrm{pH}$ 7.4) to obtain $25 \mu \mathrm{M}$ stocks and stored at $-20^{\circ} \mathrm{C}$. The same volume of PBS was added to cells in the control group. The cells were used for subsequent experimentation following 5 days of decitabine treatment at $37^{\circ} \mathrm{C}$ with $5 \% \mathrm{CO}_{2}$ in a fully humidified atmosphere.

Cell viability assay. A methyl triazolyl tetrazolium (MTT)-based assay was used to study the effect of decitabine on the viability of the expanded MDS-MSCs at P3. Briefly, cells were seeded at a density of 3,000 cells/well in 96-well plates for 1-7 days and treated with decitabine or PBS. The cell viability was measured every $24 \mathrm{~h}$ for $168 \mathrm{~h}$, MTT solution $(5 \mathrm{mg} / \mathrm{ml})$ was added and the cultures were incubated for $4 \mathrm{~h}$. The MTT-formazan crystals were dissolved overnight in $20 \%$ SDS and 50\% dimethylformamideat $\mathrm{pH} 4.7$ and absorbance was measured at a wavelength of $570 \mathrm{~nm}$.

Cell cycle assay. BM-MSCs (P2) treated with decitabine or PBS for 5 days were fixed with $70 \%(w / v)$ ice-cold ethanol overnight at $4^{\circ} \mathrm{C}$, washed twice with PBS and treated with $50 \mu \mathrm{g} / \mathrm{ml} \mathrm{RNase}$ for $30 \mathrm{~min}$ at $37^{\circ} \mathrm{C}$ followed by $10 \mu \mathrm{g} / \mathrm{ml}$ propidium iodine (PI) for $30 \mathrm{~min}$ in the dark at $4^{\circ} \mathrm{C}$. DNA content was analyzed using a flow cytometer and FlowJo software (version 10; FlowJo LLC).

Apoptosis assay. BM-MSCs (P2) were treated with decitabine or PBS for 5 days and the number of apoptotic cells was quantified using the Annexin V-FITC Apoptosis Detection kit (Invitrogen; Thermo Fisher Scientific, Inc.), according to the manufacturer's protocol. Cells were incubated 7-AAD for $30 \mathrm{~min}$ on ice. Early apoptotic cells were defined as Annexin $\mathrm{V}^{+} / 7-\mathrm{AAD}^{-}$cells, whereas late apoptotic, dead cells presented as Annexin $\mathrm{V}^{+} / 7-\mathrm{AAD}^{+}$and annexinV $/ 7-\mathrm{AAD}^{+}$, respectively. The analyses were performed using a flow cytometer and FlowJo software (version 10; FlowJo LLC).

MDS-MSCs and peripheral blood mononuclear cells (PBMCs) co-culture. MDS-MSCs (P3) were seeded in a six-well plate at a density of $1 \times 10^{5}$ cells/well on day 0 . On day 4, $10 \mu \mathrm{M}$ mitomycin $\mathrm{C}$ was added and the cells were incubated for $4 \mathrm{~h}$ at $37^{\circ} \mathrm{C}$ with $5 \% \mathrm{CO}_{2}$ in a fully humidified atmosphere. The MSCs were trypsinized and washed twice with PBS. The MSCs were seeded onto 12-well plates at a density of $2 \times 10^{4}$ cells/well. Human peripheral blood mononuclear cells (PBMCs) from healthy donor were isolated by centrifugation ( $600 \mathrm{x} \mathrm{g}$ for $15 \mathrm{~min}$ at room temperature) using Ficoll-PaquePlus density gradient (specific gravity 1.077 g/ml; Sigma-Aldrich; Merck KGaA). PBMCs were resuspended in RPMI-1640 complete medium (10\% FBS, $1 \mathrm{mM}$ L-glutamineand $100 \mathrm{U} / \mathrm{ml}$ penicillin/streptomycin; Thermo Fisher Scientific, Inc.). PBMCs $\left(2 \times 10^{5}\right.$ cells/well) were added to each well and stimulated with $5 \mu \mathrm{g} / \mathrm{ml}$ anti-CD3 (cat. no. 16-0037; 1:500; BioLegend, Inc.), $1 \mu \mathrm{g} / \mathrm{ml}$ anti-CD28 (cat. no. 16-0289; 1:200; BioLegend, Inc.) and $200 \mathrm{IU} / \mathrm{ml}$ recombinant interleukin 2 (cat. no. 200-02; 1:500; PeproTech). MDS-MSCs with treated with decitabine or PBS and PBMC cells were co-cultured at a 1:10 ratio for 5 days in RPMI-1640 complete medium at $37^{\circ} \mathrm{C}$ with $5 \% \mathrm{CO}_{2}$ in a fully humidified atmosphere prior to further experimentation.

Flow cytometry analysis. For immunophenotype analysis, the suspended cells from the co-cultures were harvested and washed with PBS containing $0.5 \%$ bovine serum albumin (BSA; Sigma-Aldrich; Merck KGaA, Darmstadt, Germany), and incubated with primary antibodies $(10-20 \mathrm{ng} / \mathrm{ml})$ for $30 \mathrm{~min}$ at $4^{\circ} \mathrm{C}$. The Human Regulatory T Cell Staining kit (cat. no. 88-8995; eBioscience; Thermo Fisher Scientific, Inc.) included primary antibodies against CD4, CD25 and forkhead box P3 (FOXP3; antibodies all 1:100). Same-species, same-isotype non-specific antibodies (cat. no. 15-4321; 1:100; eBioscience; Thermo Fisher Scientific, Inc.) were used as a 
Table II. Primer sequences for reverse transcription quantitative polymerase chain reaction.

Primer sequence (5'-3')

\begin{tabular}{lll}
\cline { 2 - 3 } Gene & \multicolumn{1}{c}{ Forward } & \multicolumn{1}{c}{ Reverse } \\
\hline Cyclin dependent kinase inhibitor 1A & ACATCTTCTGCCTTAGTCTCA & GACTAAGGCAGAAGATGTAG \\
Sp7 transcription factor & TCTCCATCTGCCTGGCTCCTT & CTGCACGCTGCCGTCAGCATG \\
Runt related transcription factor 2 & TCTTCAGCACAGTGACACCAT & CTGTTGCGCAGCCACCACCG \\
CD274 & GGTGCCGACTACAAGCGAAT & TGACTGGATCCACAACCAAAATT \\
$\beta$-actin & TATGGAGAAGATTTGGCACC & ATGAGACACACCTAAGGACC
\end{tabular}

negative control. Cell analysis was performed with FlowJo software (version 10; FlowJo LLC).

Reverse transcription-quantitative polymerase chain reaction $(R T-q P C R)$. Total RNA was extracted from $\mathrm{P} 3$ undifferentiated BM-MSCs using the RNeasy Mini kit (Qiagen GmbH, Hilden, Germany), according to the manufacturer's protocol. cDNA was prepared using the Revert Aid ${ }^{\mathrm{TM}}$ First Strand cDNA Synthesis kit (Fermentas; Thermo Fisher Scientific, Inc.), according to the manufacturer's protocol. A total of $1 \mu \mathrm{g}$ total RNA was added to $20 \mu \mathrm{l}$ total cDNA synthesis volume. qPCR was performed with a reaction volume of $20 \mu \mathrm{l}$ containing $10 \mu \mathrm{l}$ of $2 \mathrm{X}$ Real Master mix (TakaraBiotechnology Co., Ltd., Dalian, China), $0.5 \mu \mathrm{l}$ of each primer $(10 \mu \mathrm{M}), 1 \mu \mathrm{l} \mathrm{cDNA}$ and $8 \mu \mathrm{l}$ distilled water. qPCR was performed on an ABI 7500 Real-Time PCR system (Applied Biosystems; Thermo Fisher Scientific, Inc.). The thermocycling conditions were as follows: $95^{\circ} \mathrm{C}$ for $30 \mathrm{sec}$ and up to $40 \mathrm{cycles}$ of $95^{\circ} \mathrm{C}$ for $5 \mathrm{sec}$ and $60^{\circ} \mathrm{C}$ for $34 \mathrm{sec}$ according to the manufacturer's instructions. The primer sequences for runt-related transcription factor 2 (RUNX2), Sp7 transcription factor (SP7), cyclin dependent kinase inhibitor 1A (CDKN1A) and CD274 are presented in Table II. mRNA levels were quantified using the $2^{-\Delta \Delta C q}$ method (23) and normalized to the internal reference gene $\beta$-actin.

Statistical analysis. The results were statistically analyzed using SPSS software (version 20.0; IBM Corp.). The Student's t-test was used for analysis of two groups, and the one-way analysis of variance followed by the least significant difference post hoc test was used for the analysis of multiple groups. The results are presented as the mean \pm standard deviation from at least three independent experiments. $\mathrm{P}<0.05$ was considered to indicate a statistically significant difference.

\section{Results}

Cell viability of MSCs from patients with MDS is not inhibited by decitabine. The immunophenotype of the MSCs isolated from patients with MDS was detected using flow cytometry. All cell samples were positive for CD90

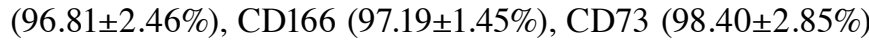
and CD105 (99.17 $\pm 1.27 \%)$ expression, but negative for CD45 $(9.79 \pm 0.89 \%)$ and CD34 (2.12 $\pm 0.99 \%)$ expression (Fig. 1A). MDS-MSCs layers were observed under light microscopy and exhibited fibroblast-like morphology (Fig. 1B). Under the appropriate differentiation conditions, MDS-MSCs were induced to differentiate into adipogenic and osteogenic cells (Fig. 1C and D).

The effect of decitabine on the viability of MDS-MSCs was investigated by exposing cells to $0.25 \mu \mathrm{M}$ decitabine and assessing the cell viability with a MTT assay. The number of live cells corresponding with the optical density of MDS-MSCs treated with decitabine was slightly lower compared with the controls over the 7-day culture period. However, no significant difference was observed between the two groups (Fig. 2A). The apoptosis assay results showed that the percentages of early apoptotic cells, late apoptotic cells and dead cells in the control group were $4.19 \pm 1.16,3.93 \pm 1.90$ and $2.33 \pm 2.95 \%$, respectively, whereas the percentages in the decitabine-treated group were $3.84 \pm 1.53,4.39 \pm 2.23$ and $3.02 \pm 3.91 \%$, respectively (Fig. 2B and C). No significant difference was observed between the control group and decitabine-treated group $(\mathrm{P}=0.618,0.664$ and 0.673 , respectively; Fig. 2D).

Decrease of the cell number in G0/lphases in MDS-MSCs treated with decitabine. The present study investigated the effects of decitabine on the cell cycle by staining the nuclei of the untreated and decitabine-treated MDS-MSCs with PI (Fig. 3A and B). Analyses of the cell cycle distribution following exposure to decitabine showed that the number of cells in the G1 phase $(84.84 \pm 5.46 \%)$ was significantly reduced in comparison with that of the PBS group (89.77\%; $\mathrm{n}=11 ; \mathrm{P}=0.023$ ) and was simultaneously accompanied by a significant increase in the number of cells in the $\mathrm{G} 2 / \mathrm{M}$ phase ( $8.71 \pm 5.65$ vs. $3.76 \pm 3.51 \% ; n=11 ; P=0.017$; Fig. 3 C). A significant difference in the number of cells in $\mathrm{S}$ phase between the two groups was not observed (6.34 \pm 2.87 vs. $6.48 \pm 2.78$; $n=11$; $\mathrm{P}=0.910$; Fig. 3C). In line with these findings, the gene expression of CDKN1A, a key mediator of senescence (19), was slightly lower in the decitabine-treated group compared with the control group. Accordingly the $\Delta \mathrm{Cq}$ value of CDKN1A in the decitabine-treated group $(4.86 \pm 1.35)$ was slightly higher compared with the control group (3.97 \pm 0.98$)$. However, no significant difference was observed between the two groups $(n=10 ; P=0.054 ;$ Fig. 3D). The results obtained suggested that the senescent phenotype of MDS-MSCs seems to be partly ameliorated by decitabine treatment.

Osteogenic differentiation of MDS-MSCs is not significantly affected by decitabine. The effect of decitabine on the ability of MDS-MSCs to differentiate into an osteogenic lineage was investigated. The results demonstrated that the $\Delta \mathrm{Cq}$ values of 
A
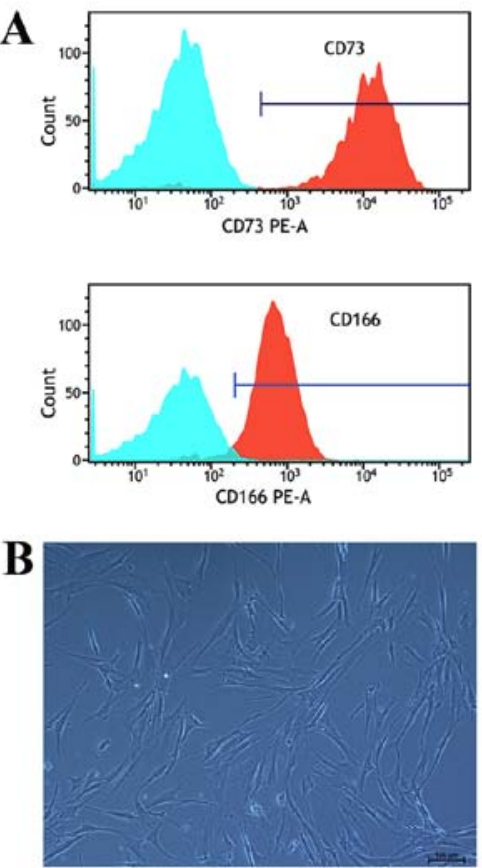
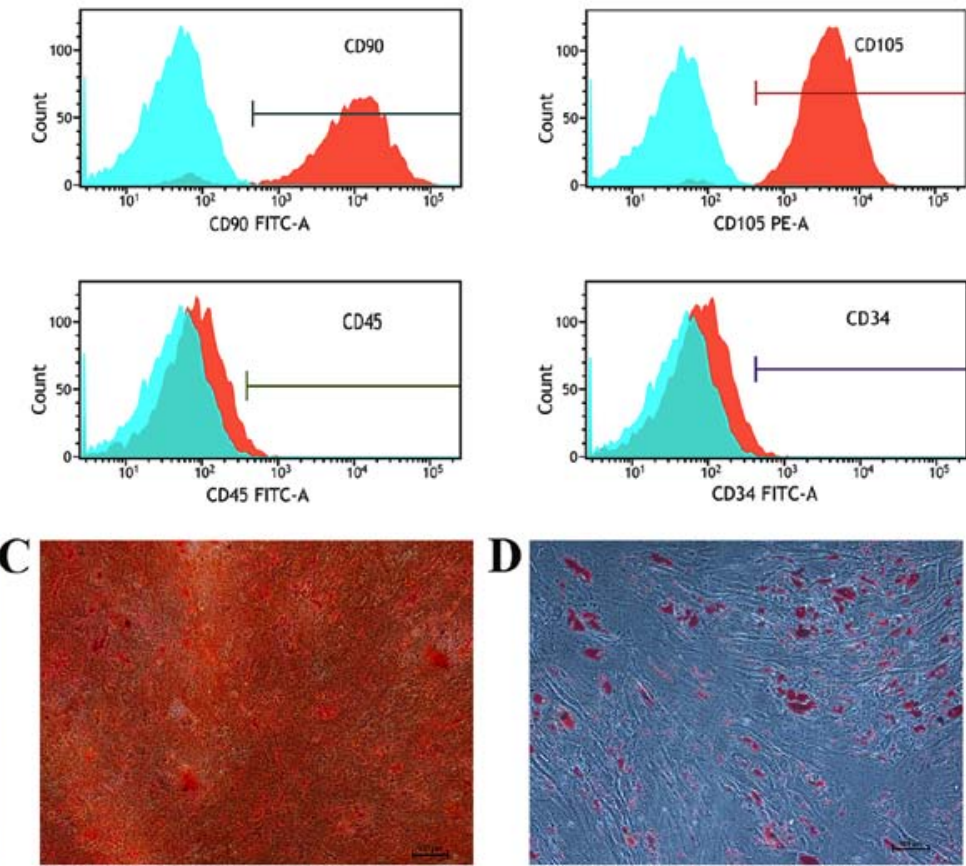

Figure 1. Morphology, immunophenotypic characteristics and differentiation potential of bone marrow-MSCs from patients with MDS. (A) The expression of MSC positive (CD73, CD90, CD105 and CD166) and negative (CD34 and CD45) markers. The light blue represents the respective isotype control, while the red represents MDS-MSCs. (B) MDS-MSCs exhibited fibroblast-like morphology. (C) Alizarin Red S staining of MSCs cultured in the osteogenic induction media for 14 days. (D) Oil Red O staining of cells cultured in the adipogenic induction media for 14 days. Original magnification, x100. MSC, mesenchymal stem cell; MDS, myelodysplastic syndrome.
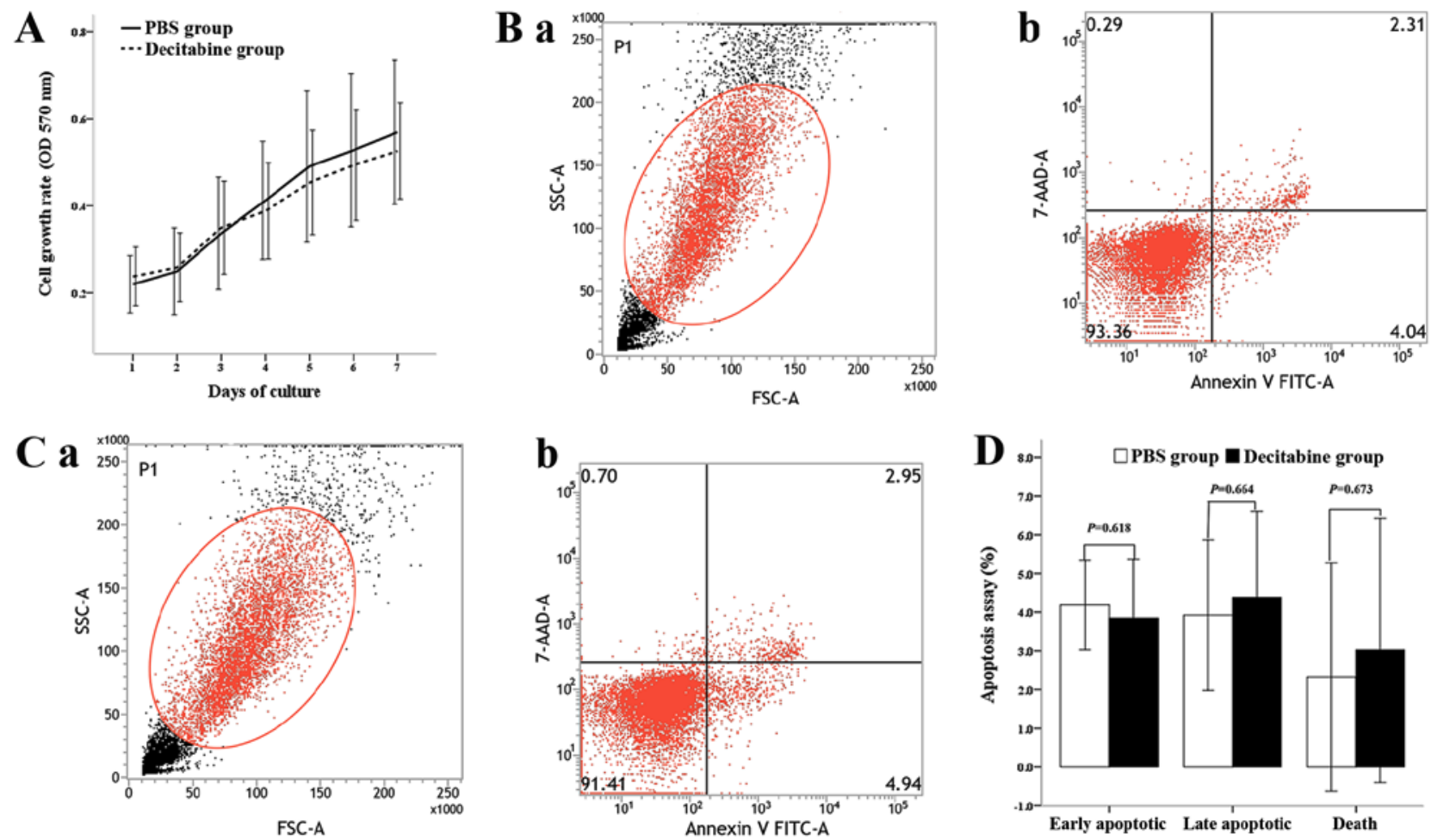

Figure 2. Effect of viability on the proliferation and apoptosis of BM-MSCs from patients with myelodysplastic syndromes. (A) Growth curve of BM-MSCs was plotted using an MTT assay. The absorbance represents the viability of MDS-MSCs. Decitabine-treated BM-MSCs grew more slowly compared with the control group $(\mathrm{n}=10)$. The apoptosis rate was measured by flow cytometry in the control and decitabine-treated groups. (B) (a) The FSC/SSC gating-graph for the control group. The cells in the red circle represent the cells that were analyzed. (b) Early apoptotic cells of control group. (C) (a) The FSC/SSC gating-graph for decitabine-treated MDS-MSCs. The cells in the red circle represent the cells that were analyzed. Early apoptotic cells, late apoptotic cells and dead cells were defined as Annexin V-positive/7AAD-negative, Annexin V-positive/7AAD-positive, and Annexin V-negative/7AAD-positive cells, respectively. (b) Early apoptotic cells in the decitabine-treated group. (D) The y-axis represents percentages of the cells in each group. No significant difference in the percentage of early apoptotic, late apoptotic and dead cells was observed between the decitabine-treated and control groups ( $\mathrm{n}=8$ ). Student's t-test was used to analyze statistically significant differences. All data are presented as the mean \pm standard deviation. BM-MSCs, bone marrow mesenchymal stem cells; FSC, forward scatter; SSC, side scatter; 7ADD, 7-aminoactinomycin D; FSC, forward scatter; SSC, side scatter; FITC, fluorescein isothiocyanate; OD, optical density. 
A
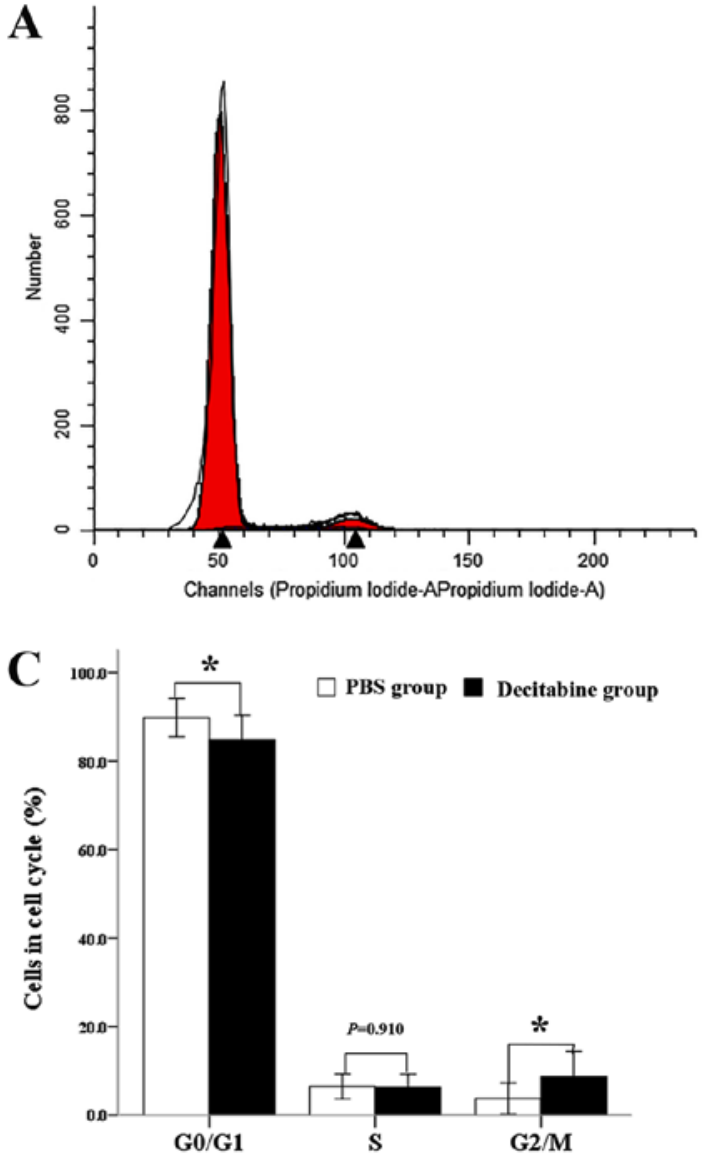

B

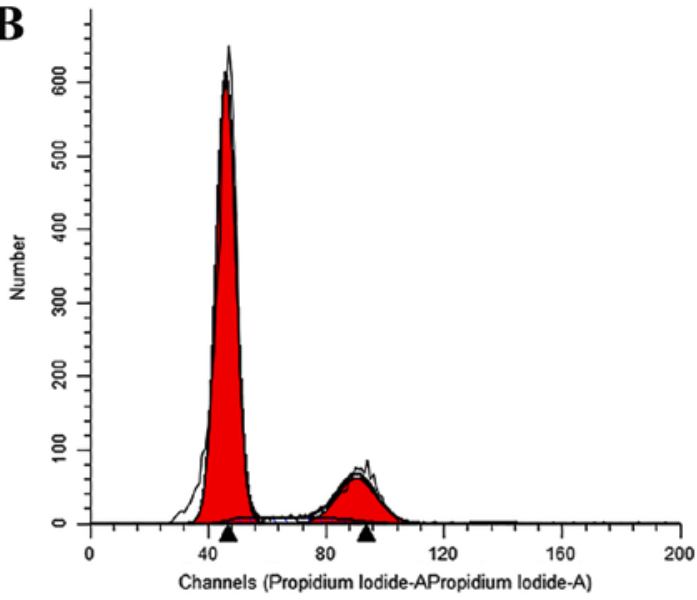

D

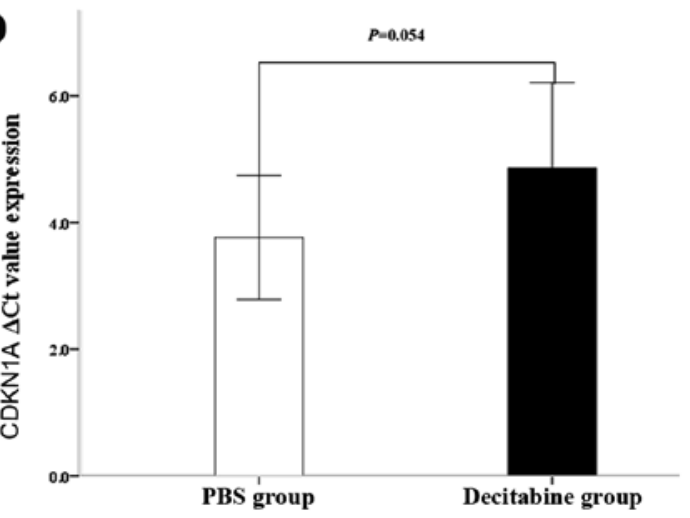

Figure 3. Effect of decitabine on the cell cycle distribution of BM-MSCs. The cells were exposed to $0.25 \mu \mathrm{M}$ decitabine for 5 days. The BM-MSCs were collected, fixed and stained with propidium iodide for flow cytometry analysis. The DNA content is represented on the X-axis, the number of cells counted is represented on the y-axis. (A) The cell cycle distribution of the control group. (B) The cell cycle distribution of the decitabine-treated group. (C) The graph indicates the percentages of cells in the G0/G1, S and G2/M phases of the cell cycle. A reduction in G0/G1 phase and an increase in G2/M phase was observed for BM-MSCs of the decitabine group compared with the control group (P<0.05). (D) The expression of CDKN1A in the PBS group and decitabine group was measured by reverse transcription-quantitative polymerase chain reaction. The $\Delta \mathrm{Cq}$ value of CDKN1A in the decitabine-treated group was higher compared with the control group. The black arrowheads on the represent the peak values of G0/1 and S phases, respectively. The results are expressed as means \pm standard deviation. Student's t-test was used to detect statistically significant differences. ${ }^{*} \mathrm{P}<0.05$. BM-MSCs, bone marrow mesenchymal stem cells; CDKN1A, cyclin dependent kinase inhibitor 1A.

RUNX2 in MDS-MSCs without osteogenic induction were not significantly different between the two groups $(3.67 \pm 1.22$ for the decitabine-treated group; $3.15 \pm 1.46$ for the control group; $\mathrm{n}=15 ; \mathrm{P}=0.303$; Fig. $4 \mathrm{~A})$. The $\Delta \mathrm{Cq}$ value of $\mathrm{SP} 7$ slightly decreased in the decitabine-treated group $(5.92 \pm 2.15)$ compared with the control group $(6.00 \pm 1.46)$ but was not statistically significant ( $\mathrm{n}=15 ; \mathrm{P}=0.909 ;$ Fig. 4B).

Immunosuppressive phenotype of MDS-MSCs is reduced by decitabine treatment. In the present study, decitabine-or PBS-treated MDS-MSCs were co-cultured with PBMCs in vitro. The results indicated that decitabine-and $\mathrm{PBS}$-treated MDS-MSCs efficiently generated $\mathrm{CD} 4^{+} \mathrm{CD} 25^{+} \mathrm{FOXP} 3^{+}$Tregs from activated $\mathrm{T}$ cells compared with $\mathrm{PBMCs}$ alone $(2.00 \pm 1.77 \%$ for PBMCs, $3.94 \pm 1.55 \%$ for the PBS group and $3.96 \pm 2.42 \%$ for the decitabine group, $\mathrm{P}=0.015$ and $\mathrm{P}=0.014$, respectively; Fig. 5A and $\mathrm{B}$ ). The effect of decitabine on the ability of MDS-MSCs to induce T cells into Tregs was investigated. The results showed that the induction of Tregs cell was decreased in nine out of 13 decitabine-treated MDS-MSCs samples. In the aforementioned nine samples of MDS-MSCs, the percentage of $\mathrm{CD}^{+}{ }^{+} \mathrm{T}$ cells was similar between the decitabine group and the PBS group (34.20 \pm 7.39 vs. $34.43 \pm 6.14 \% ; \mathrm{P}=0.944)$. Furthermore, the percentage of $\mathrm{CD} 4{ }^{+} \mathrm{CD} 25^{+}$cells was not significantly different between the decitabine and PBS groups $(4.27 \pm 2.33$ vs. $4.03 \pm 2.89 \%$; $\mathrm{P}=0.849$ ). The induction of $\mathrm{T}$ cells to Tregs was lower in the decitabine-treated group compared with the PBS group (decitabine group, 2.56 $\pm 0.93 \%$; PBS group, $4.30 \pm 1.57 \%$; $\mathrm{n}=9 ; \mathrm{P}=0.019$; Fig. 5C). To minimize the effect of the two groups of MDS-MSC on $\mathrm{CD}^{+}{ }^{+} \mathrm{T}$ cells, the ratios of Tregs to $\mathrm{CD}^{+} \mathrm{T}$ cells in the two groups were calculated. The percentage of the decitabine group was lower compared with that of the PBS group (7.68 \pm 3.12 vs. $12.69 \pm 5.55 \% ; \mathrm{P}=0.031$; Fig. 5D).

As CD274 on MSCs serves an important role in inducing activated $\mathrm{T}$ cells to differentiate into Tregs (24), the effect of decitabine treatment on the expression levels of CD274 on MDS-MSCs was investigated in the current study. The $\Delta \mathrm{Cq}$ value of CD274 on the MDS-MSCs in the decitabine-treated group was significantly increased compared with the control group $(12.36 \pm 0.32$ vs. $11.24 \pm 0.91 ; \mathrm{P}=0.010)$, indicating that the expression of CD274 on MDS-MSCs was inhibited by decitabine. 

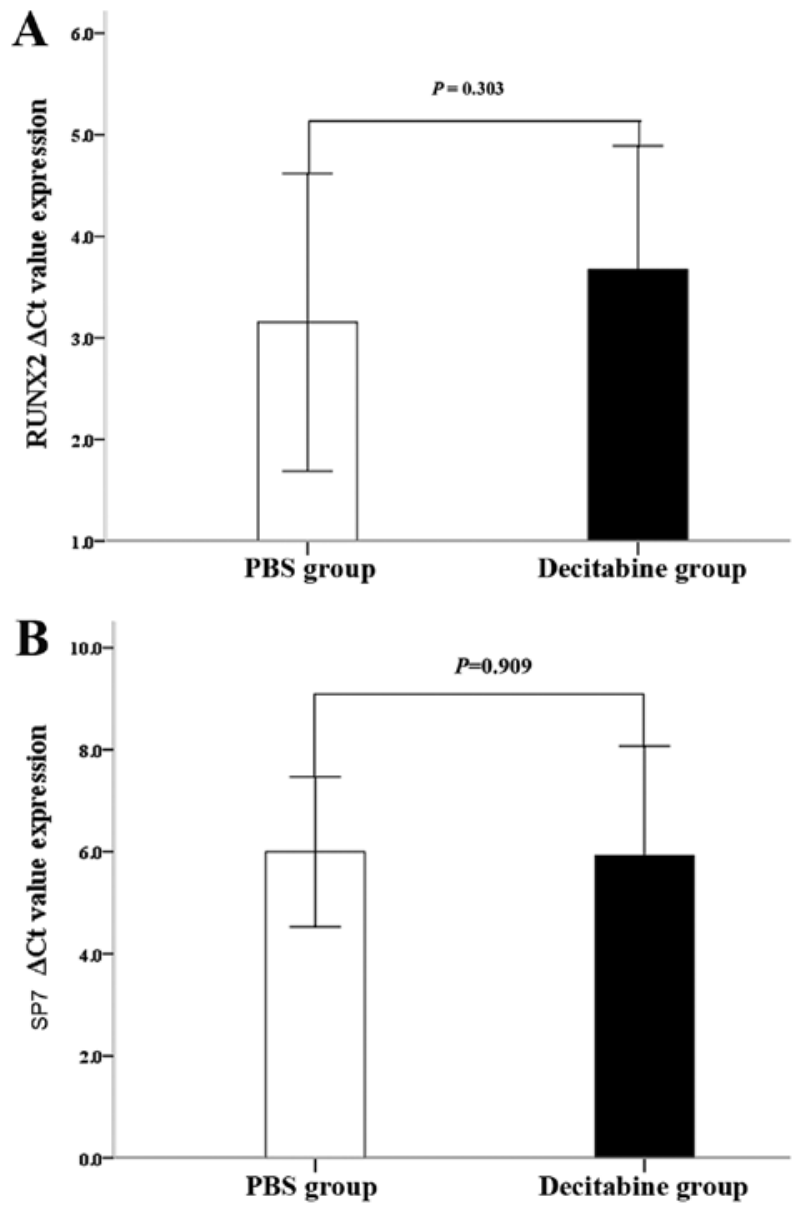

Figure 4. Effect of decitabine on the gene expression associated with the osteoblastic differentiation in bone marrow mesenchymal stem cells. Reverse transcription-quantitative polymerase chain reaction was performed to detect the expression levels of the osteogenic differentiation-associated genes RUNX2 and SP7 before osteogenic induction. The effect of decitabine on the mRNA relative expression levels of (A) RUNX2and (B) SP7 were not statistically significant $(\mathrm{P}>0.05)$. The results are expressed as means \pm standard deviation. The Student's t-test was used to detect statistically significant differences. RUNX2, runt related transcription factor 2; SP7, Sp7 transcription factor.

\section{Discussion}

MDS is a complex disease that involves the hematopoietic and BM microenvironment (4-6). Previous studies demonstrated that the primary dysfunction of MSCs may contribute to the evolution of MDS $(5,6)$. The role of MDS-MSCs in the pathogenesis of MDS remains unclear; however, the senescent phenotype of MDS-MSCs may be involved in the progression of MDS and may be a potential therapeutic target (4). Therefore, the present study investigated the effect of decitabine on the senescent phenotype of MDS-MSCs.

The results obtained indicated that there was no significant effect on the growth potential, apoptosis and mRNA expression levels of transcription factors involved in the osteogenic differentiation of MDS-MSCs treated with decitabine compared with the control group. However, an altered cell cycle was detected in MDS-MSCs treated with decitabine; notably a decrease in the number of cells at the G1 phase and an increase in the number of cells at the G2/M phase compared with the control group. In addition, decitabine significantly diminished the induction of activated T cells to differentiate into Tregs by MDS-MSCs, which was associated with the decreased expression of PD-L1 on decitabine-treated MDS-MSCs compared with the control group.

A previous pharmacokinetic study revealed that decitabine exhibits in vitro antitumor effects on primary human leukemia cells at concentrations $(0.02-0.30 \mu \mathrm{M})$ similar to which tumor cells in responding patients with MDS/AML are likely exposed to in clinical settings (20). Previous studies have also demonstrated that $0.10-0.25 \mu \mathrm{M}$ decitabine can induce an immune response $(21,25)$. Therefore, $0.25 \mu \mathrm{M}$ decitabine was selected to study effects on MSCs in the present study. However, other studies on solid tumor cell lines revealed that the growth of tumor cells was not significantly inhibited by decitabine at such low doses $(26,27)$. Decitabine at low doses may not cause cytotoxicity and DNA damage, but rather induces transcription of endogenous dsRNAs in ovarian cancer that activate the viral recognition and interferon response pathway, which lead to a tumor-inhibiting immune response $(14,28)$. The current study revealed that the mRNA levels of SP7 and RUNX2, the transcription factors involved in the early differentiation process toward osteoblasts (10), were not affected by $0.25 \mu \mathrm{M}$ decitabine. These results indicated that the mRNA expression levels of the transcription factors involved in osteogenic differentiation in MDS-MSCs in the undifferentiated state were not affected by decitabine treatment. Previous studies revealed that these transcription factors induced the MSCs into hematopoietic tissue and bone, i.e. a donor-derived ectopic bone marrow, through a cartilage intermediate in the adult mouse kidney capsule (29,30). Furthermore, certain patients with MDS responded to decitabine treatment (22). Thus further studies are required to clarify such discrepancies. It is important to consider the dosage of decitabine when interpreting the results obtained in the current study. However, other mechanisms including the post-transcriptional regulation of the transcription factors, DNA methylation and miRNAs, may be involved in the actions of decitabine $(9,10)$.

The current study investigated the effect of decitabine on the senescent phenotype of the isolated MDS-MSCs. The cellular senescence was associated with cells in the G0/1 phase of the cell cycle (31). The results obtained in the current study indicated that the cell cycle of MDS-MSCs was altered by decitabine treatment, leading to fewer cells in the G0/G1 phases. A previous study revealed that the senescent phenotype of MDS-MSCs was associated with cells accumulated in the G0/1 phase of the cell cycle (9). Accordingly, the mRNA expression level of CDKN1A in MDS-MSCs treated with decitabine was lower compared with the control group; however, this difference was not statistically significant. It is likely due to the fact that CDKN1A is not the only G0/G1 phase regulator; there are several other molecules, including TP53 and miRNAs, which are involved in the process $(9,19)$.

Tregs expansion in malignant diseases leads to the suppression of host antitumor responses and is a feature of the progression to aggressive subtypes in MDS (32). Tregs serve an important role during the progression of MDS from a low to a high-risk stage. Previous studies reported that the ability of high-risk MDS-MSCs to induce T cells to differentiate into Tregs is greater compared with low-risk MDS-MSCs $(32,33)$. The current study showed that he 

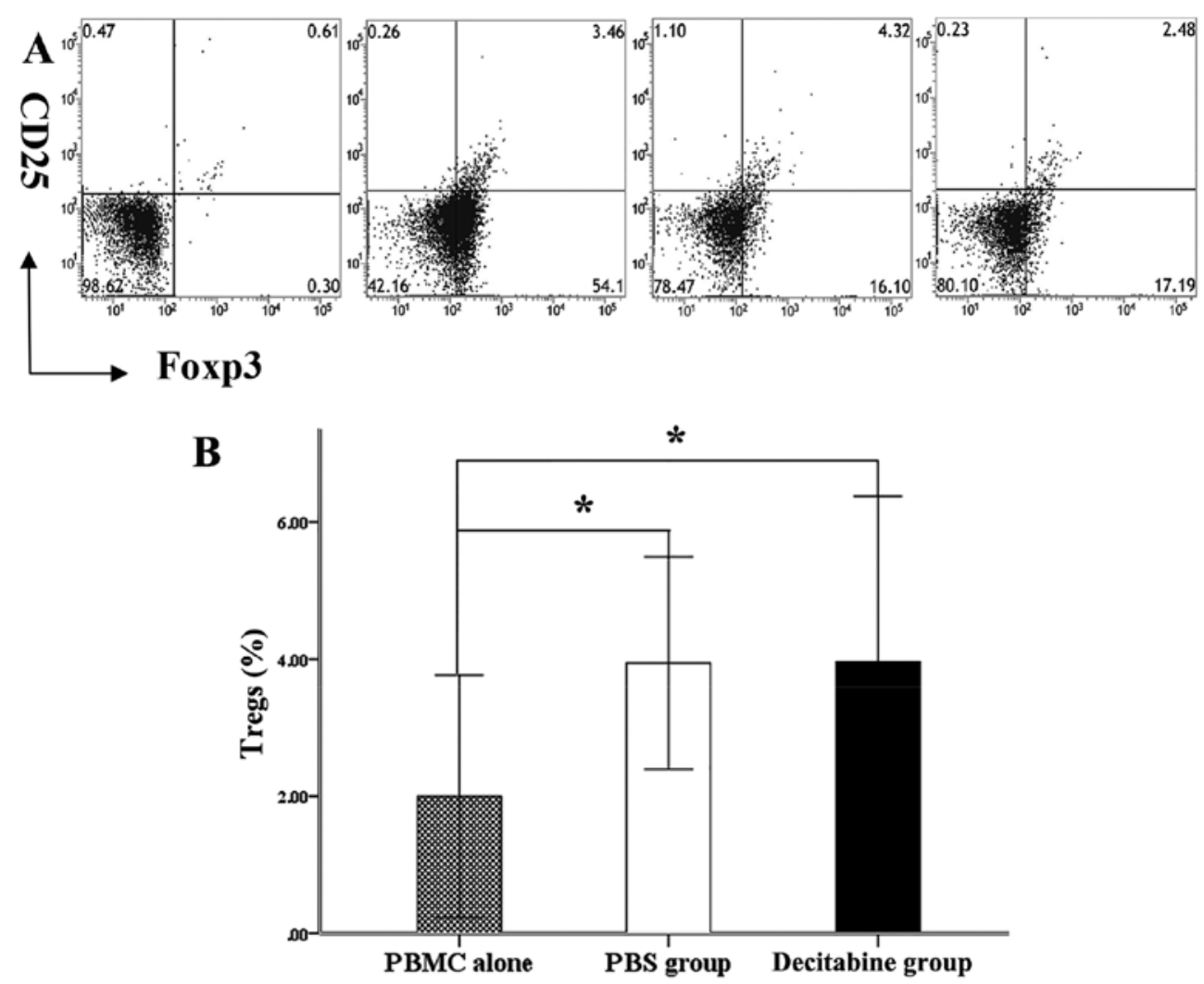

$\mathbf{C}$

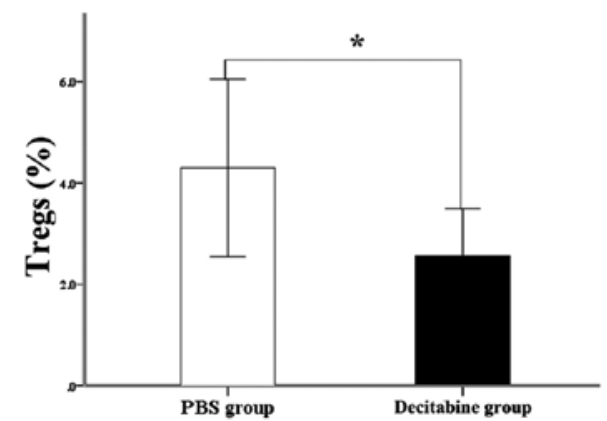

D

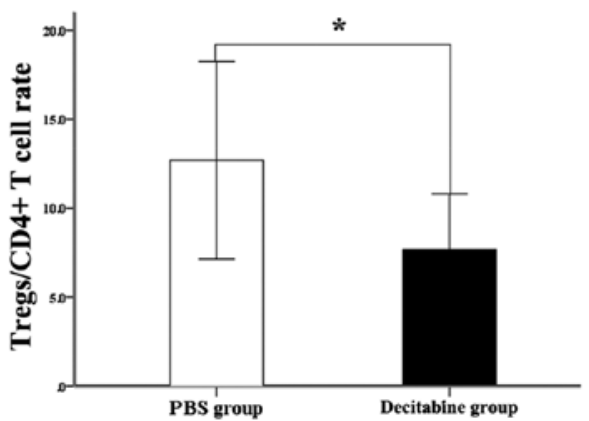

Figure 5. Effect of decitabine on the immunomodulatory function of MDS-MSCs. For the detection of Tregs, forward vs. side scatter was plotted for lymphocytes, followed by gating for $\mathrm{CD} 4^{+} \mathrm{T}$ cells. These cells were then analyzed for the expression of CD25 and FoxP3. (A) The expression of CD25 and Foxp3 on $\mathrm{CD} 4^{+} \mathrm{T}$ cells was analyzed by flow cytometry. (B) Both decitabine-treated and PBS-treated MDS-MSCs efficiently generated $\mathrm{CD} 4^{+} \mathrm{CD} 25^{+} \mathrm{Foxp} 3^{+} \mathrm{Tregs}$ from activated $\mathrm{T}$ cells. The results are expressed as means $\pm \mathrm{SD}$. The least significant difference test was used to detect statistically significant differences. (C) The Tregs induction in the decitabine group was significantly lower compared with that in the PBS group. Results are expressed as mean \pm SD of nine separate experiments. (D) The ratio of the percentage of Tregs to $\mathrm{CD} 4^{+} \mathrm{T}$ cells $\left(\mathrm{CD} 4^{+} \mathrm{CD} 25^{+} \mathrm{FOXP} 3^{+} / \mathrm{CD} 4^{+}\right)$in the decitabine group was significantly decreased compared with the control group. The results are expressed as means $\pm \mathrm{SD}$. Student's t-test was used to detect statistically significant differences. $\mathrm{P}<0.05$. MDS-MSCs, myelodysplastic syndromes-mesenchymal stem cells; Tregs, regulatory T cells; Foxp3, CD, cluster of differentiation; forkhead box P3; SD, standard deviation.

induction of activated $\mathrm{T}$ cells to differentiate into Tregs was impeded by MDS-MSCs treated with $0.25 \mu \mathrm{M}$ decitabine. The aforementioned results suggested that decitabine treatment reverses the inhibitory immune microenvironment induced by MDS-MSCs. These results have important clinical implications, as the immunosuppressive microenvironment created by the senescent MSCs serves an important role in the progression from low-to high-risk MDS (33). The decreased expression of CD274 on decitabine-treated MDS-MSCs may be the mechanism underlying the impaired ability of the MDS-MSCs to induce activated T cells to differentiate into Tregs. CD274, a main ligand for programmed cell death 1 , is widely expressed in tissues and contributes to the immunosuppressive microenvironment in MDS that protects malignant cells from immune destruction (34). Reversing the cellular senescence of MDS-MSCs may delay the progression of MDS (9).

The results of the current study suggested that MDS-MSCs may be targeted by novel pharmacological intervention and has important clinical implications. In recent years, knowledge surrounding immunological dysfunction and the function of the HSC niche in MDS has increased. A cure for MDS and 
AML is unlikely to be achieved through the selective targeting of the dysplastic/leukemic clones. Rather, a combination approach consisting of the following approaches may be more effective: i) targeting of the malignant clones; ii) targeting of the immune cells, with the aim of releasing the suppressive microenvironment or reconstruction of the antitumor immune response; and iii) modulating the surrounding microenvironmental protective niche to render it less hospitable to malignant cells and more amenable to normal HSCs (35).

In summary, the current study revealed that $0.25 \mu \mathrm{M}$ decitabine altered the cell cycle distribution of the isolated MSCs from patients with MDS. Furthermore, decitabine-treated MDS-MSCs inhibited the induction of activated $\mathrm{T}$ cells to differentiate into Treg cells. The results obtained in the current study suggested that MDS may be partly ameliorated by reducing the immunosuppressive function of MDS-MSCs by decitabine treatment, providing a novel avenue for MDS interventions.

\section{Acknowledgements}

Not applicable.

\section{Funding}

The current study was supported by grants from the National Natural Science Foundation of China (grant no. 81500102), the Science and Technology Planning Project of Guangdong (grant nos. 2014B020212009, 2014B020226002, 2015B020227003, 2015B020226001 and 2017B020230004), the Science and Technology Planning Project of Guangzhou (grant nos. 201803040005, 201803040011 and 201400000003-4) and the Natural Science Foundation of Guangdong (grant no. S2013030013305).

\section{Availability of data and materials}

The datasets used and/or analyzed during the current study are available from the corresponding author on reasonable request.

\section{Authors' contributions}

SG, HZ, PLL, JW, and XD conceived the study and designed the experiments. SG, HZ, PJL, LZ, ZL, and JW performed the experiments. SG, PLL, LZ, and JW analyzed the data. SG, JW and XD prepared the paper. YP performed the experiments, analyzed the data and co-wrote the manuscript. All authors read and approved the final manuscript.

\section{Ethics approval and consent to participate}

MDS-MSCs from patients with MDS and Human PBMCs from health individual were obtained with informed consent for research purposes, and the procedures were approved by the Ethics Committees of Guangdong General Hospital.

\section{Patient consent for publication}

Consent to publish has been obtained from the participant to report individual patient data.

\section{Competing interests}

The authors declare that they have no competing interests.

\section{References}

1. Adès L, Itzykson R and Fenaux P: Myelodysplastic syndromes. Lancet 383: 2239-2252, 2014.

2. Shastri A, Will B, Steidl U and Verma A: Stem and progenitor cell alterations in myelodysplastic syndromes. Blood 129: 1586-1594, 2017.

3. Ferrer RA, Wobus M, List C, Wehner R, Schönefeldt C, Brocard B, Mohr B, Rauner M, Schmitz M, Stiehler M, et al: Mesenchymal stromal cells from patients with myelodyplastic syndrome display distinct functional alterations that are modulatedbylenalidomide. Haematologica 98: 1677-1685, 2013.

4. Medyouf H, Mossner M, Jann JC, Nolte F, Raffel S, Herrmann C, Lier A, Eisen C, Nowak V, Zens B, et al: Myelodysplastic cells in patients reprogram mesenchymal stromal cells to establisha transplantable stem cell niche disease unit. Cell Stem Cell 14: 824-837, 2014.

5. Zambetti NA, Ping Z, Chen S, Kenswil KJG, Mylona MA, Sanders MA, Hoogenboezem RM, Bindels EMJ, Adisty MN, Van Strien PMH, et al: Mesenchymal inflammation drives genotoxic stressin hematopoietic stem cells and predicts diseaseevolution in human pre-leukemia. Cell Stem Cell 19: 613-627, 2016.

6. Raaijmakers MH, Mukherjee S, Guo S, Zhang S, Kobayashi T, Schoonmaker JA, Ebert BL, Al-Shahrour F, Hasserjian RP, Scadden EO, et al: Bone progenitor dysfunction induces myelodysplasia andsecondary leukemia. Nature 464: 852-857, 2010.

7. Wang Y, Chen X, Cao W and Shi Y: Plasticity of mesenchymal stem cells inimmunomodulation: Pathological andtherapeutic implications. Nat Immunol 15: 1009-10016, 2014.

8. Bagley RG, Weber W, Rouleau C, Yao M, Honma N, Kataoka S, Ishida I, Roberts BL and Teicher BA: Human mesenchymal stem cells from bone marrow express tumor endothelial and stromal markers. Int J Oncol 34: 619-627, 2009.

9. Zhao Y, Wu D, Fei C, Guo J, Gu S, Zhu Y, Xu F, Zhang Z, Wu L, Li X and Chang C: Down-regulation of Dicer1 promotes cellular senescence and decreases the differentiation and stem cell-supporting capacities of mesenchymal stromal cells in patients with myelodysplastic syndrome. Haematologica 100: 194-204, 2015.

10. Geyh S, Oz S, Cadeddu RP, Fröbel J, Brückner B, Kündgen A, Fenk R, Bruns I, Zilkens C, Hermsen D, et al: Insufficient stromal support in MDS results from molecular andfunctional deficits of mesenchymal stromal cells. Leukemia 27: 1841-1851, 2013.

11. Pang Y, Deng C, Geng S, Weng J, Lai P, Liao P, Zeng L, Lu Z, Zhang $J$ and Du X: Premature exhaustion of mesenchymal stromal cellsfrom myelodysplastic syndrome patients. Am J Transl Res 9: 3462-3468, 2017.

12. Pavlaki K, Pontikoglou CG, Demetriadou A, Batsali AK, Damianaki A, Simantirakis E, Kontakis M, Galanopoulos A, Kotsianidis I, Kastrinaki MC and Papadaki HA: Impaired proliferative potential of bone marrow mesenchymal stromal cells in patients with myelodysplastic syndromes is associated with abnormal wnt signaling pathway. Stem Cells Dev 23: 1568-1581, 2014.

13. Falconi G, Fabiani E, Fianchi L, Criscuolo M, Raffaelli CS, Bellesi S, Hohaus S, Voso MT, D'Alò F and Leone G: Impairment of PI3K/AKT and WNT/ $\beta$-catenin pathways in bone marrow mesenchymal stem cells isolated from patients with myelodysplastic syndromes. Exp Hematol 44: 75-83.e1-4, 2016.

14. Roulois D, Loo Yau H, Singhania R, Wang Y, Danesh A, Shen SY, Han H, Liang G, Jones PA, Pugh TJ, et al: DNA-demethylating agents target colorectal cancer cells by inducing viral mimicry by endogenous transcripts. Cell 162: 961-973, 2015.

15. Chiba N, Furukawa K, Takayama S, Asari T, Chin S, Harada Y, Kumagai G, Wada K, Tanaka T, Ono A, et al: Decreased DNA methylation in the promoter region of the WNT5A and GDNF genes may promote the osteogenicity of mesenchymal stemcells from patients with ossified spinal ligaments. J Pharmacol Sci 127: 467-473, 2015.

16. Teklemariam T,Purandare B,Zhao L and Hantash BM: Inhibition of DNA methylation enhances HLA-G expression in human mesenchymal stem cells. Biochem Biophys Res Commun 452: 753-759, 2014. 
17. Vardiman JW, Thiele J, Arber DA, Brunning RD, Borowitz MJ, Porwit A, Harris NL, Le Beau MM, Hellström-Lindberg E, Tefferi A and Bloomfield CD: The 2008 revision of the World Health Organization (WHO) classification ofmyeloid neoplasms and acute leukemia: Rationale and important changes. Blood 114 937-951. 2009

18. Dominici M, Le Blanc K, Mueller I, Slaper-Cortenbach I, Marini F, Krause D, Deans R, Keating A, Prockop DJ and Horwitz E: Minimal criteria for defining multipotent mesenchymal stromal cells. The international society for cellular therapy position statement. Cytotherapy 8: 315-317, 2006.

19. Fei C, Zhao Y, Guo J, Gu S, Li X and Chang C: Senescence of bone marrow mesenchymal stromal cells is accompanied by activation of p53/p21 pathway inmyelodysplastic syndromes. Eur J Haematol 93: 476-486, 2014.

20. Tsai HC, Li H, Van Neste L, Cai Y, Robert C, Rassool FV, Shin JJ, Harbom KM, Beaty R, Pappou E, et al: Transient low doses of DNA-demethylating agents exert durable antitumor effects on hematological and epithelial tumor cells. Cancer Cell 21: 430-446, 2012.

21. Yao Y, Zhou J, Wang L, Gao X, Ning Q, Jiang M, Wang J, Wang L and Yu L: Increased PRAME-specific CTL killing of acute myeloid leukemia cells by either a novel histone deacetylase inhibitor chidamide alone or combined treatment with decitabine. PLoS One 8: e70522, 2013.

22. Ding K, Fu R, Liu H, Nachnani DA and Shao ZH: Effects of decitabine on megakaryocyte maturation in patients with myelodysplastic syndromes. Oncol Lett 11: 2347-2352, 2016.

23. Livak KJ and Schmittgen TD: Analysis of relative gene expression data using real-time quantitative PCR and the 2(-Delta Delta $\mathrm{C}(\mathrm{T}))$ method. Methods 25: 402-408, 2001.

24. Armand P: Immune checkpoint blockade in hematologic malignancies. Blood 125: 3393-3400, 2015.

25. Chiappinelli KB, Strissel PL, Desrichard A, Li H, Henke C, Akman B, Hein A, Rote NS, Cope LM, Snyder A, et al: Inhibiting DNA methylation causes an interferon response in cancer via dsRNA including endogenous retroviruses. Cell 169: 361, 2017.

26. Shin DY, Sung Kang H, Kim GY, Kim WJ, Yoo YH and Choi YH Decitabine, a DNA methyltransferases inhibitor, induces cell cycle arrest at G2/M phase through p53-independent pathway in human cancer cells. Biomed Pharmacother 67: 305-311, 2013.

27. Shang D, Han T, Xu X and Liu Y: Decitabine induces G2/M cell cycle arrest by suppressing $\mathrm{p} 38 / \mathrm{NF}-\mathrm{\kappa B}$ signaling inhuman renal clear cell carcinoma. Int J Clin Exp Pathol 8: 11140-11148, 2015.
28. Diesch J, Zwick A, Garz AK, Palau A, Buschbeck M and Götze KS: A clinical-molecular update onazanucleoside-based therapy for thetreatment of hematologic cancers. Clin Epigenetics 8: 71, 2016.

29. Reinisch A, Etchart N, Thomas D, Hofmann NA, Fruehwirth M, Sinha S, Chan CK, Senarath-Yapa K, Seo EY, Wearda T, et al: Epigenetic and in vivo comparison of diverse MSC sources reveals an endochondral signature for human hematopoietic niche formation. Blood 125: 249-260, 2015.

30. Chan CK, Chen CC, Luppen CA, Kim JB, DeBoer AT, Wei K, Helms JA, Kuo CJ, Kraft DL and Weissman IL: Endochondral ossification is required for haematopoietic stem-cell niche formation. Nature 457: 490-494, 2009.

31. Mao Z, Ke Z, Gorbunova V and Seluanov A: Replicatively senescent cells are arrested in G1 and G2 phases. Aging (Albany NY) 4: 431-435, 2012.

32. Kordasti SY, Ingram W, Hayden J, Darling D, Barber L, Afzali B, Lombardi G, Wlodarski MW, Maciejewski JP, Farzaneh F and Mufti GJ: CD $4^{+} \mathrm{CD} 25$ high Foxp3 $3^{+}$regulatory T cells in myelodysplastic syndrome. Blood 110: 847-850, 2007.

33. Zhao Z, Wang Z, Li Q, Li W, You Y and Zou P: The different immunoregulatory functions of mesenchymal stem cells in patients with low-risk or high-risk myelodysplastic syndromes. PLoS One 7: e45675, 2012.

34. Yang H, Bueso-Ramos C, DiNardo C, Estecio MR, Davanlou M, Geng QR, Fang Z, Nguyen M, Pierce S, Wei Y, et al: Expression of PD-L1, PD-L2, PD-1 and CTLA4 in myelodysplastic syndromes is enhanced by treatment with hypomethylating agents. Leukemia 28: 1280-1288, 2014.

35. Pleyer L, Valent $P$ and Greil R: Mesenchymal stem and progenitor cells in normaland dysplastic hematopoiesis-masters of survivaland clonality? Int J Mol Sci 17: pii: E1009, 2016.

This work is licensed under a Creative Commons Attribution-NonCommercial-NoDerivatives 4.0 International (CC BY-NC-ND 4.0) License. 